\title{
An Intrarenal Adrenocortical Carcinoma Arising in an Adrenal Rest
}

\author{
Ji Hee Lee Y Young Deuk Choi $^{1}$ \\ Nam Hoon Cho
}

Departments of Pathology and 'Urology, Severance Hospital, Yonsei University College of Medicine, Seoul, Korea

Received: May 23, 2018

Revised: July 2, 2018

Accepted: July 20, 2018

\section{Corresponding Author}

Nam Hoon Cho, MD

Department of Pathology, Severance Hospital,

Yonsei University College of Medicine, 50-1

Yonsei-ro, Seodaemun-gu, Seoul 03722, Korea

Tel: +82-2-2228-1767

Fax: +82-2-2227-7939

E-mail:CH01988@yuhs.ac
We describe a case of a 61-year-old Korean man who was diagnosed with renal cell carcinoma that was discovered on abdominopelvic computed tomography obtained after the patient complained of back pain. A radical nephrectomy was performed, and the surgical specimen showed a relatively well-circumscribed and yellowish lobulated hard mass. Microscopically, the tumor showed sheets and nests of hypercellular pleomorphic cells with thick fibrous septation, frequent mitoses, and areas of adrenal cortical-like tissue. Immunohistochemical staining revealed that the tumor cells were positive for inhibin- $\alpha$, vimentin, synaptophysin, and melan A. It also revealed that the tumor cells were negative for pan-cytokeratin, epithelial membrane antigen, paired box 8 , $\alpha$-methylacyl-coenzyme A racemase, CD10, cytokeratin 7, carbonic anhydrase 9, c-Kit, renal cell carcinoma, transcription factor E3, human melanoma black 45, desmin, smooth muscle actin, S-100, chromogranin A, CD34, anaplastic lymphoma kinase, and integrase interactor 1. Based on these histopathological and immunohistochemical findings, we diagnosed the tumor as intrarenal adrenocortical carcinoma arising in an adrenal rest. Several cases of intrarenal adrenocortical carcinoma have been reported, although they are very rare. Due to its poor prognosis and common recurrence or metastasis, clinicians and pathologists must be aware of this entity.

Key Words: Adrenocortical carcinoma; Adrenal rest tumor; Carcinoma; Renal cell
Adrenocortical carcinoma is a rare and heterogeneous malignancy with a poor prognosis, the pathogenesis of which is not yet completely understood. Patients present with hormone excess or local mass effect. ${ }^{1}$ An adrenal rest (ectopic adrenal tissue) can occur anywhere along the gonadal descent. This tissue usually has no clinical significance, but it may become hyperplastic or malignant in patients with primary or secondary adrenal pathology. ${ }^{2}$ Most adrenal rest tumors are functional and diagnosed preoperatively. However, the less frequent nonfunctional adrenal rest tumors are discovered accidentally or postoperatively. ${ }^{3}$ We described a patient with a nonfunctioning intrarenal adrenocortical carcinoma that arose from an adrenal rest.

\section{CASE REPORT}

This study was approved by the Institutional Review Board of Severance Hospital with a waiver of informed consent (IRB No. 4-2017-1044).

A 61-year-old man was evaluated for back pain that had persisted for 10 days. Abdominopelvic computed tomography (APCT) and magnetic resonance imaging were performed at a local clinic and revealed a $13-\mathrm{cm}$ mass in his right kidney. The mass was causing an ureteropelvic junction obstruction that broadly contacted the second and third duodenal portions, psoas muscle, and inferior vena cava. All radiologic findings were consistent with renal cell carcinoma with multiple lung metastases. The patient was hospitalized at our institution, and the APCT was repeated and provided the same diagnosis (Fig. 1A).

The patient underwent radical nephrectomy without adrenalectomy. The right kidney weighed $1,135 \mathrm{~g}$ and measured $17 \mathrm{x}$ $12 \times 6 \mathrm{~cm}$. The tumor had a smooth and bulging external surface. Cross sections revealed a well-circumscribed and yellowish lobulated hard mass (Fig. 1B) measuring $14 \times 12 \times 8 \mathrm{~cm}$, present in the mid pole of the right kidney. The mass showed extensive necrosis $(60 \%)$ and hemorrhage (30\%).

Microscopically, the tumor had multilobulated nests divided by thick fibrous septations (Fig. 2A). The tumor was comprised of compact polygonal cells with distinct cell borders and granular cytoplasm. Sinusoidal vascular ingrowth was less distinct. Nuclei were round or ovoid, hyperchromatic with central prominent nucleoli, and contained frequent mitoses (40/50 high-power fields) (Fig. 2B) without raisinoid nuclei or perinuclear haloes. Some areas containing adrenal cortical-like tissues were identified (Fig. 2C). Necrosis and vascular invasion were also present. These 

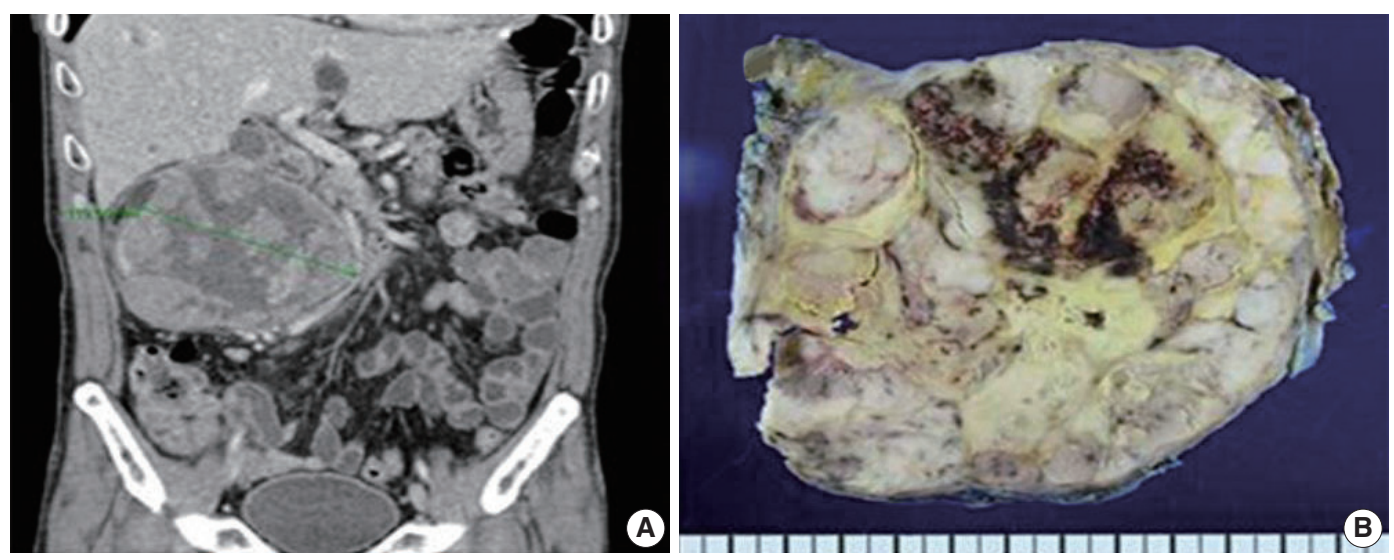

Fig. 1. Imaging and gross findings of adrenocortical carcinoma. (A) Abdominopelvic computed tomography reveals a $13 \mathrm{~cm}$ heterogeneous mass in the right kidney. (B) Bisected kidney specimen showing a lobulated hard mass with necrosis and hemorrhage.
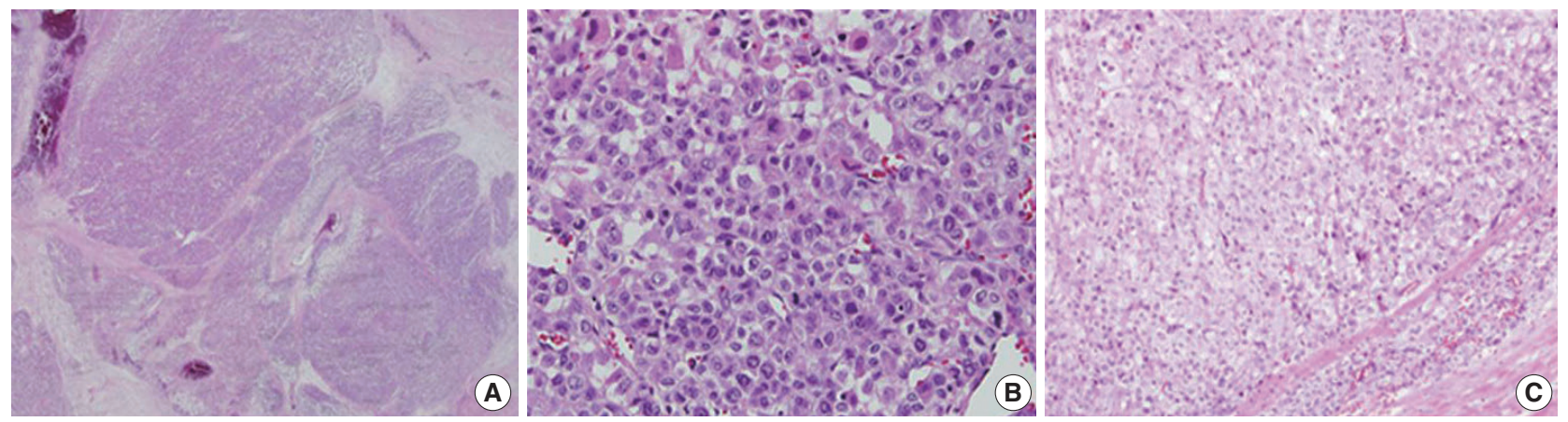

Fig. 2. Histologic findings of adrenocortical carcinoma. (A) Sheets and nests of hypercellular tumor cells with thick fibrous septation. (B) Pleomorphic cells with frequent mitoses. (C) Areas of adrenal cortical-like tissue.

results suggested against the diagnosis of renal cell carcinoma.

Immunohistochemical staining results are presented in Table 1 and Fig. 3. The results were consistent with adrenocortical carcinoma and excluded the possibility of renal cell carcinoma and specific variants of the kidney tumor.

\section{DISCUSSION}

Ectopic or accessory adrenal cells are often found postnatally along the path of gonadal descent because the adrenocortical primordium develops in close proximity to the urogenital ridge of the emerging gonad and migrates alongside the gonad. ${ }^{4}$ Typically, these cells disappear within a few years of birth, and sometimes these cells linger without any event.

Ye et al. ${ }^{4}$ reported seven cases of intrarenal adrenal tissue and two cases of renal-adrenal fusion. Except for one case identified within the kidney mid pole, all intrarenal lesions were found in the superior portion of the kidney. Our case reports a malignant tumor arising from the ectopic adrenal rest in the mid pole of the kidney. In all nine reported cases of Ye et al., ${ }^{4}$ the intrarenal
Table 1. Immunostaining results of adrenocortical carcinoma

\begin{tabular}{ll}
\hline Antigen & Tumor cell \\
\hline Pan-cytokeratin & Negative \\
EMA & Negative \\
Inhibin- $\alpha$ & Positive \\
Vimentin & Positive \\
Melan A & Focal positive \\
Synaptophysin & Positive \\
Paired box 88 & Negative \\
Calretinin & Negative \\
$\alpha$-Methylacyl-coenzyme A racemase & Negative \\
CD10 & Negative \\
Cytokeratin 7 & Negative \\
Carbonic anhydrase 9 & Negative \\
C-kit & Negative \\
Renal cell carcinoma & Negative \\
Transcription factor E3 & Negative \\
Human melanoma black 45 & Negative \\
Desmin & Negative \\
Smooth muscle actin & Negative \\
S-100 & Negative \\
Chromogranin A & Negative \\
CD 34 & Negative \\
Anaplastic lymphoma kinase & Negative \\
Integrase interactor 1 & No loss \\
\hline &
\end{tabular}



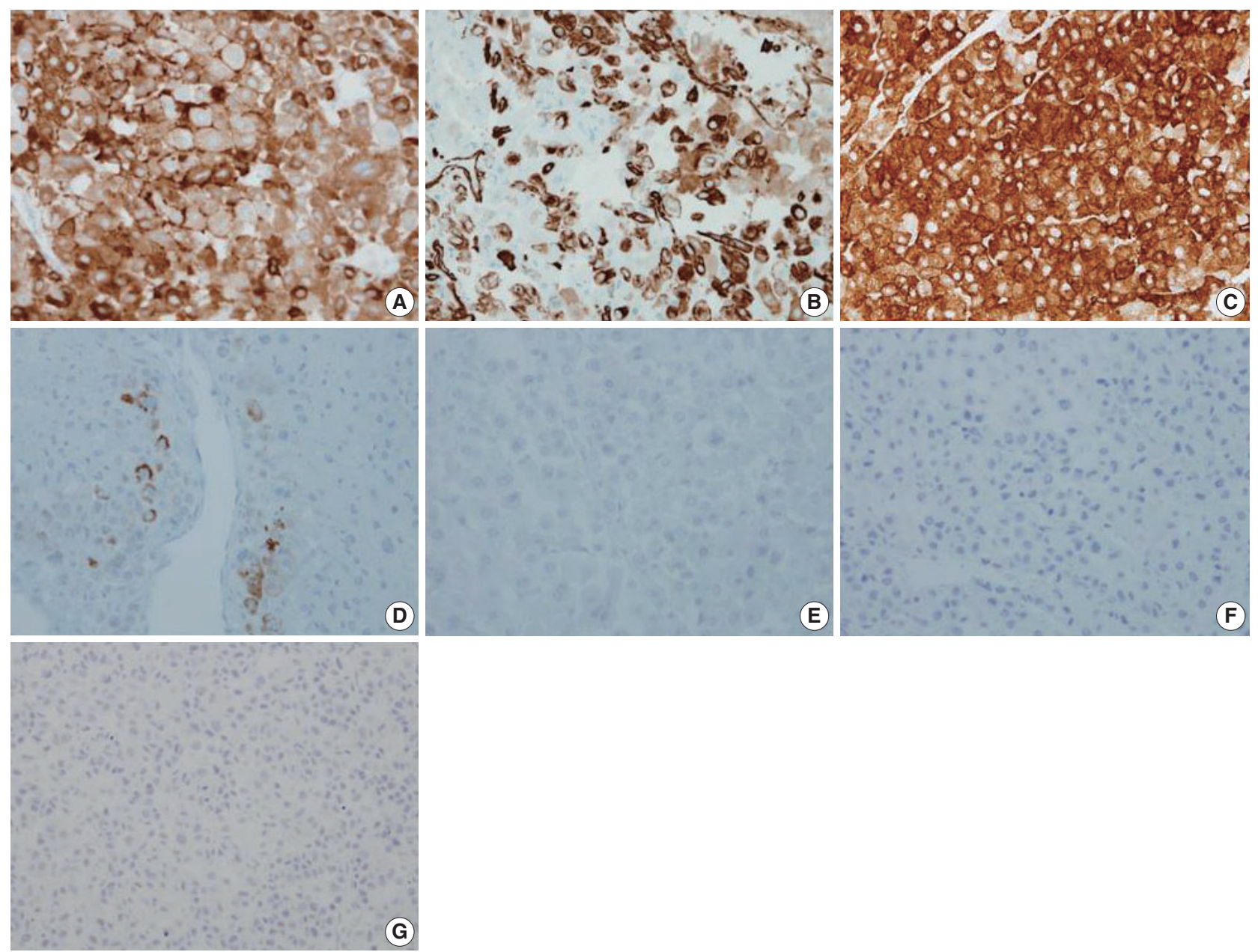

Fig. 3. Immunohistochemical staining results of adrenocortical carcinoma. The tumor cells were positive for inhibin- $\alpha(A)$, vimentin (B), synaptophysin (C), and melan A (D) and negative for epithelial membrane antigen (E), paired box $8(\mathrm{~F})$, and pan-cytokeratin (G).

adrenal tissues were composed of only adrenal cortical tissue with no adrenal medullary tissue present. There is a recent review of the literature about adrenocortical carcinoma arising in an adrenal rest. Reported malignant tumors arising from an ectopic adrenal rest are predominantly adrenocortical carcinomas of the retroperitoneum, gonad, liver, kidney, spinal cord, and pelvis. ${ }^{5}$ Intrarenal adrenocortical carcinomas have been previously identified in the hilum ${ }^{3}$ and in the mid pole of the kidney as in our case. Adrenocortical carcinomas that involve the gonads show relatively high rates of mortality. ${ }^{6-8}$

The microscopic features that favor the diagnosis of renal cell carcinoma over adrenocortical carcinoma are the presence of glands, particularly if they contain red blood cells, and abundant cytoplasmic glycogen. However, neither is pathognomonic and were present in the case. Among the nine histological parameters of the Weiss scoring system for histologic diagnosis of adrenocortical carcinoma (high nuclear grades [Fuhrman nuclear grades III and
IV], mitotic rate $>5 / 50$ high-power fields, atypical mitotic figures, clear tumor cell cytoplasm [less than $25 \%$ tumor cells], diffuse architecture [greater than 33\% of tumor], necrosis, venous invasion, sinusoidal invasion, and capsular invasion), the present case met seven parameters, excluding sinusoidal invasion and capsular invasion. After the diagnosis of renal cell carcinoma was excluded by morphology and negative cytokeratin expression, we examined additional immunohistochemical stains to differentiate epithelioid angiomyolipoma, adrenocortical carcinoma, glomus tumor, or related mesenchymal tumors. Finally, we defined the tumor as adrenocortical carcinoma (pT2NxcM1).

Currently, radical surgery is the only curative approach, and it is recommended for all patients with resectable adrenocortical carcinoma tumors, including those patients with recurrent disease. There is no consensus concerning adjuvant therapy. ${ }^{9}$ However, recent studies have reported that adjuvant mitotane may prolong recurrence-free survival in patients with radically resected adre- 
nocortical carcinoma. $^{10,11}$

Our patient was treated with radical nephrectomy and adjuvant chemotherapy (VAP; vincristine, doxorubicin, and prednisolone) with mitotane. He has been healthy with no evidence of recurrence or metastasis for 3 months after the original diagnosis. Recent studies have reported the prevalence of adrenocortical carcinoma in Korea to be $2 \%-5 \%$. In our institution, five cases of adrenocortical carcinoma were reported from 2000 to 2018. Among them, this is the only and first reported case of intrarenal adrenocortical carcinoma. We reported a rare case of intrarenal adrenocortical carcinoma arising from an ectopic adrenal rest as a mimicker of renal cell carcinoma in the kidney. Although the incidence of malignancy arising in an adrenal rest is low, clinicians and pathologists must be aware of the possibility because of its poor prognosis and common recurrence and metastasis.

\section{ORCID}

Ji Hee Lee: https://orcid.org/0000-0003-0054-7033

Nam Hoon Cho: https://orcid.org/0000-0002-0045-6441

\section{Conflicts of Interest}

No potential conflict of interest relevant to this article was reported.

\section{REFERENCES}

1. Allolio B, Fassnacht M. Clinical review: adrenocortical carcinoma: clinical update. J Clin Endocrinol Metab 2006; 91: 2027-37.

2. Barwick TD, Malhotra A, Webb JA, Savage MO, Reznek RH. Embryology of the adrenal glands and its relevance to diagnostic imaging. Clin Radiol 2005; 60: 953-9.

3. Goren E, Engelberg IS, Eidelman A. Adrenal rest carcinoma in hilum of kidney. Urology 1991; 38: 187-90.

4. Ye H, Yoon GS, Epstein JI. Intrarenal ectopic adrenal tissue and renaladrenal fusion: a report of nine cases. Mod Pathol 2009; 22: 175-81.

5. Cornejo KM, Afari HA, Sadow PM. Adrenocortical carcinoma arising in an adrenal rest: a case report and review of the literature. Endocr Pathol 2017; 28: 165-70.

6. Engel FL, McPherson HT, Fetter BF, et al. Clinical, morphological and biochemical studies on a malignant testicular tumor. J Clin Endocrinol Metab 1964; 24: 528-42.

7. Morimoto Y, Hiwada K, Nanahoshi M, et al. Cushing's syndrome caused by malignant tumor in the scrotum: clinical, pathologic and biochemical studies. J Clin Endocrinol Metab 1971; 32: 201-10.

8. Jain SH, Sadow PM, Nosé V, Dluhy RG. A patient with ectopic cortisol production derived from malignant testicular masses. Nat Clin Pract Endocrinol Metab 2008; 4: 695-700.

9. Langer P, Bartsch D, Moebius E, Rothmund M, Nies C. Adrenocortical carcinoma: our experience with 11 cases. Langenbecks Arch Surg 2000; 385: 393-7.

10. Terzolo M, Angeli A, Fassnacht M, et al. Adjuvant mitotane treatment for adrenocortical carcinoma. N Engl J Med 2007; 356: 2372-80.

11. Berruti A, Grisanti S, Pulzer A, et al. Long-term outcomes of adjuvant mitotane therapy in patients with radically resected adrenocortical carcinoma. J Clin Endocrinol Metab 2017; 102: 1358-65. 